\title{
Microfluidic Impedance Biosensors For Monitoring A Single And Multiple Cancer Cells In Anticancer Drug Treatments
}

\author{
T. A. Nguyen ${ }^{1}$, Tien V. Nguyen ${ }^{1}$, D.T. Tran ${ }^{2}$, Toan V. Nguyen ${ }^{1}$, C.H. Le ${ }^{3}$, V.B. Nguyen ${ }^{4}$ and H.Q. Le \\ ${ }^{1}$ Le Quy Don Technical University, Ha Noi, Viet Nam \\ ${ }^{2}$ VNU University of Engineering and Technology, Ha Noi, Vietnam \\ ${ }^{3}$ Faculty of Engineering and Science, University of Greenwich, Kent, United Kingdom \\ ${ }^{4}$ College of Engineering and Technology, University of Derby, Derby, United Kingdom \\ ${ }^{5}$ Saigon Hi-Tech Park - SHTP, Ho Chi Minh, Vietnam
}

\begin{abstract}
In this work, we present a novel microfluidic impedance biosensor chip for trapping both a single and multiple cancer cells and monitoring their response to the anticancer drug treatment. By designing different sizes of working microelectrodes together with the $\mathrm{V}$ shaped cell capture structures, a single or multiple cells are trapped on the microelectrodes surfaces. In addition, by utilizing the passive pumping method, cells can be trapped and positioned inside the microchannels without the need of using the outer micro pump or syringe. The impedance change induced by the response of cells to the anticancer drug Cisplatin treatment was successfully recorded. The proposed biosensor chip has a great potential for applications in cancer cell research, drug screening, and quantification of cancer cells from various tumor stages. The results of this study open potential research collaborations about development of costeffective devices and lab-on-chips for early disease detection, studies of cancerous cells and their response to anti-cancer drugs to optimize cancer treatments, characterisation of mechanical properties of cells, new drug delivery mechanisms, and micro and nano manufacturing.
\end{abstract}

Keywords - Microfluidic, Biosensor, Impedance, Single cell, Cancer, Anticancer drug treatment.

\section{INTRODUCTION}

Cell-based impedance biosensors have been recognized as valuable and powerful tools for detecting biochemical effects such as cellular physiological changes [1], pharmaceutical effects [2], and environmental toxicities [3]. It can be used to study various cellular activities in a real-time, label-free and nondestructive manner, including cell spreading, growth, and motility; this is done via monitoring the electrical alternations at the interfaces between the cell and electrode [4,5]. For conventional cell-based sensors, a large cell population is normally used, and they are randomly seeded on the top of big-sized electrodes, due to their lim- ited ability to trap and control a single cell $[2,6]$. Therefore, the average measurement is assumed to represent the behavior of a typical cell within a cell population. This might lead to the inaccuracy or misleading results [7]. Therefore, there has been an emerging demand to develop innovative and smart devices which are able to be used to study the behaviors and signals from a single cell.

Recently, the fast advancements of microfluidic techniques as well as micro and nano manufacturing brought many advantages for single cell studies $[8,9]$. By owning unique features such as a small size, laminar flow, and small volumes of samples and reagents, chip-based microfluidics have attracted the growing attention about a single cell monitoring and analysis. The proposed microfluidic sensor chip in this work can capture a single and multiple breast cancer MCF-7 cells on the microelectrode surface for monitoring the sequential cellular behavior and testing the anticancer drug.

The rest of the paper is organized as follows. Section II presents design and fabrication of a chip and experiments. Section III discusses the main results and challenges of using microfluidics for single cell studies. Finally, Section IV presents conclusions and addresses the potential collaborations in Biomedical Engineering (BME), especially among research institutions in Vietnam and UK.

\section{MATERIALS AND METHOD}

\section{A. Microfluidic chip design and fabrication process}

The microfluidic impedance biosensor comprises three main parts, including the sensing, the cell capture structures, and the microfluidic. Fig. 1 presents a packaged chip with the sensing and cell trap structures. By utilizing the electrical cell-substrate impedance sensing technique [10], the sensing part composes of Microelectrode Arrays (MEAs) which are patterned in two identical channels (see Fig.1 (b)). In each channel, the MEAs comprises four columns of a working microelectrode (WE) which are located symmetrically on two sides of a rectangular large counter electrode $\left(\mathrm{CE}, 350 \times 500 \mu \mathrm{m}^{2}\right)$. Two columns of the WEs on one side 
of the CE are shifted by $40 \mu \mathrm{m}$ in order to increase the cell trapping effectiveness from the cell suspension flow. The WEs are in a square of $25 \times 25 \mu \mathrm{m}$ and $60 \times 60 \mu \mathrm{m}$ to host single cells and multiple cells respectively.

The cell capture structures are designed and arranged corresponding to the each working microelectrode. Each cell-trap composes of two identical blocks placing closely together to form a V-shaped recess. There are small gaps between these two blocks to allow the hydrodynamic flow passing through and avoiding the captured cells. The novel design of the V-shaped cell-trap in this work leads to the higher cell trapping efficiency. In addition, it can overcome the limitations of conventional hydrodynamic cell trapping methods [11].

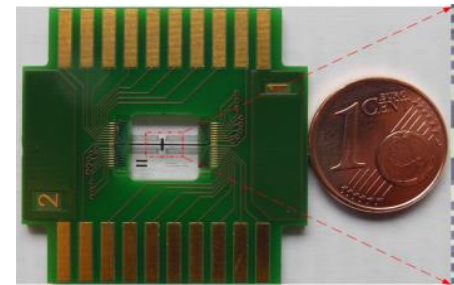

(a)

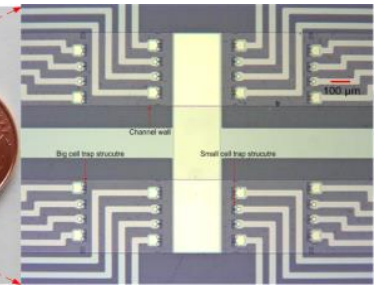

(b)
Fig.1 (a): An image of a packaged chip. (b): A micrograph of a Microelectrode Array with the 3D cell capture structures

There are two different size of trap corresponding to the size of the WEs which aim to capture single or multiple cells on the top of microelectrodes. The V-shaped traps on two sides of the CE are placed oppositely to each other and their recesses are arranged toward the inlets for trapping cells from the cell suspension flow. As a result, the microelectrodes on one side of the sensor are able to capture cells and serve as WEs, while the microelectrodes on the other side without capturing cells serve as reference microelectrodes. The microelectrode arrays are integrated inside a microfluidic channel.

The sensor was fabricated on the Pyrex wafers based on the standard micro fabrication techniques. The V-shaped cell-capture arrays are made of SU-8 of $20 \mu \mathrm{m}$ in height and fabricated in the same layer with the microchanel walls. The microfluidic part of the sensor chip consists of (1) microchannel walls with the same height as the cell capture and (2) a PDMS cover which was fabricated separately using micro moulding method [12]. Two microchannels with the height of $20 \mu \mathrm{m}$ were created inside the cover. Four holes with a diameter of $1 \mathrm{~mm}$ were punched through the PDMS layer to produce the inlets and outlets. Two microchannels of $40 \mu \mathrm{m}$ in height were formed by a natural adhesion of the PDMS cover onto the microchannel walls. The design and fabrication of a proposed sensor chip can be found in details in our previous works $[11,13]$.
After dicing, the total size of one chip is only $11 \times$ $5.3 \mathrm{~mm}^{2}$. The chip was glued into a double-sided printed circuit board (PCB) and wire bonding for the impedance measurement. By perforating a window through the PCB together with the use of a thin gold microelectrode and PDMS cover, the sensor chip is total "transparent". Therefore, it is ideal for monitoring cells inside the microchannels during experiments.

\section{B. Cell culture}

Human breast cancer cells MCF-7 were cultivated in the DMEM medium supplemented with $10 \%$ fetal bovine serum (FBS) under the standard conditions $\left(37^{\circ} \mathrm{C}, 5 \% \mathrm{CO}_{2}\right)$ inside an incubator. Cells were detached from the culture flasks by a treatment with trypsin-EDTA for $2 \mathrm{~min}$. After a detachment, they were resuspended in the DMEM to inactivate any remaining trypsin activities. After a centrifugation for $10 \mathrm{~min}$, they were resuspended in the $\mathrm{CO}_{2}$ independent medium which is supplemented with $4 \mathrm{mM} \mathrm{L}$-glutamine to the final concentration of $10^{6} \mathrm{cells} / \mathrm{ml}$.

\section{Impedance measurement}

The spectrum measurement was carried out by using Solartron impedance analyser 1260 (SI 1260). The SI 1260 delivered an alternating voltage of $10 \mathrm{mV}$ amplitude over a frequency range from $10^{2}$ to $10^{6} \mathrm{~Hz}$. For the real-time measurement, the SI 1260 was set to deliver an alternating voltage of $10 \mathrm{mV}$ at $4 \mathrm{kHz}$.

\section{Experiment procedure}

The detail of an experiment procedure can be found in our previous works [11]. Briefly, the wire bonded chip is firstly cleaned and modified. Then, the full chip is formed by aligning the PDMS cover on to the microchannel walls. Next, the cell suspended medium is injected into the channel by placing a small drop on the inlet. Single and multiple cells are captured on the top of microelectrodes. Finally, the further tasks of an experiment are able to be carried out.

\section{RESULTS}

\section{A. Hydrodynamic trapping single and multiple cells}

Figure 2 presents a photo of a chip used in the experiment (a) and a micrograph of two microelectrodes with a single and multiple cells on the top (b). Only one microchanel was used in each experiment. The chip can be reused several times by removing the PDMS cover and cleaning the chip surface. After the cells were trapped on the top of the working microelectrodes, two drops of a cell culture 
medium was placed on the inlet and the outlet. Then, the chip was placed into an incubator to culture cells. As shown in Fig. 2 (a), two working microelectrodes with cells were trapped and incubated. Two cells on the big-sized microelectrode spread and their shapes were clearly changed while a shape of a single cell on the smaller one is unchanged. The measured spectra therefore vary correspondingly.

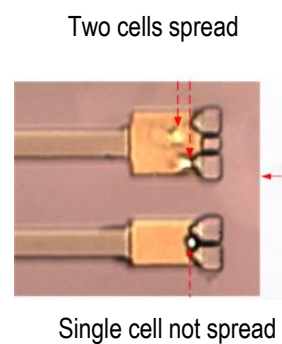

(a)

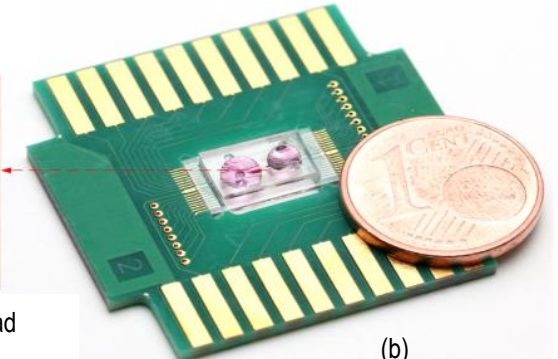

(b)
Fig.2 (a): A photo of a chip used in the experiment. (b): A micrograph of two microelectrodes with a single and multiple cells on top

\section{B. Monitoring the response of cell to anticancer drug}

Figure 3 describes the response of a single MCF-7 cell to the well-known anti-cancer drug-Cisplatin with a concentration of $100 \mu \mathrm{M}$ after exposing for 6 hours.

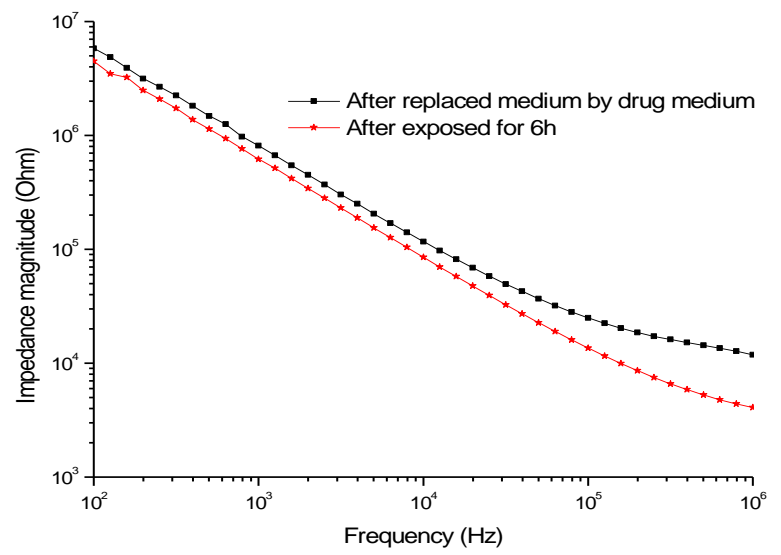

Fig.3 A Bode plot to monitor responses of a single cancer cell in the Cisplatin treatment: concentration of $100 \mu \mathrm{M}$ after exposing for 6 hours.

After trapping the cells on the top of the microelectrodes, the cells were cultured inside an incubator for 8 hours. Then, the medium inside the microchannels was replaced by physiological conditions. As shown in Fig.3, the impedance magnitude after exposing for 6 hours (RED line) sharply decreases, in comparison with the impedance magnitude, just after replacing the medium inside the microchannel by a drug medium (BLACK line).

\section{Real-time monitoring the response of cells to anticancer drug treatment}

The real-time monitoring of the response of the MCF-7 cells to the anticancer drug Cisplatin treatment is illustrated in Fig. 4. After a cell trapping process, the cells were cultured for 8 hours, so that the cells stabilise, attach, and spread on the microelectrode surface. Then, the medium inside the chip was replaced by Cisplatin to expose the cells to physiological conditions. The impedance measurement was performed for 6 hours, and the impedance magnitude decrease approximately $15 \%$.

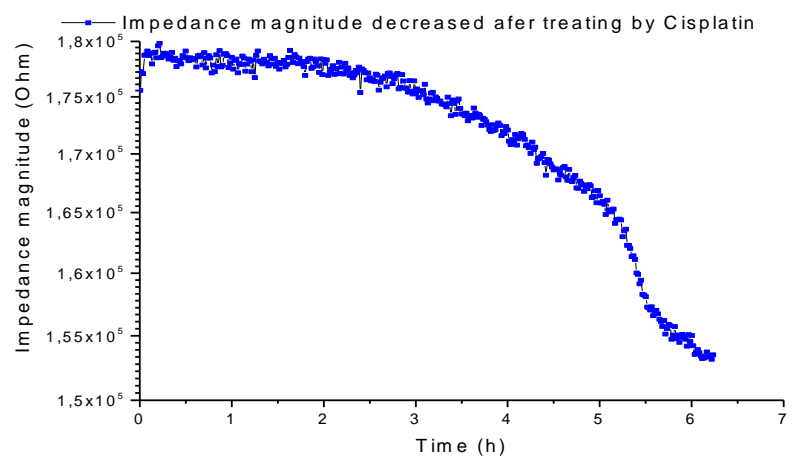

Fig. 4 A real-time monitoring of the response of the MCF-7 cell in the Cisplatin treatment with the same concentration.

\section{DiscUSSIONS AND CONCLUSIONS}

A successful development of a novel microfludic impedance biosensor which is suitable for cell-based experiments and studies is presented in this paper. The chip can trap a single or multiple cells on the surface of microelectrodes with a high efficiency for subsequent investigations. After capturing and culturing inside the microchannels, cell behaviors and their response to a surrounding environment or anticancer drug treatments can be evaluated. The chip can provide the information of a single cell in comparison with multiple cells. However, the influence on cell behaviors due to the miniature environment and the behaviors of cells in the long-term requires further tests. The results of this study have potentials for applications in the cancer cell research, drug screening, and quantification of cancer cells from various tumor stages, especially for the further research and development of biosensors and lab-on-chips which are based on behaviors and signals from cells.

Cancer is a global problem that accounts for almost $13 \%$ of deaths worldwide; and more than half of all cancer cases and nearly $2 / 3$ rd of global cancer deaths occur in developing countries; concretely 12.7 million new cancers were diagnosed worldwide in 2008, and 7 million of which were in developing countries $[14,15,17]$. By 2020 , there will be 
between 15 and 17 million new cases of cancer every year, $60-70 \%$ new cases of cancer and nearly $70 \%$ of cancer deaths will be in economically disadvantaged countries [17]. Annually, there are nearly 125 thousand people which diagnosed with cancers in Viet Nam. However, cancer is potentially the most preventable disease; with current resources, one-third of tumors could be preventable; and onethird of newly diagnosed cancer patients could experience increased survival or early-stage detection [17]. There is an urgent need for a multidisciplinary approach to improve cancer care and reduce the rates of cancer deaths in resource-poor countries in which there exist a lack of access to cancer therapy, poor early detections of cancers and screening services, unfriendly health care and delivery systems, poor organization of supportive-care facilities [16, 17]. Developments of the cost-effective solutions, including smart biosensors and lab-on-chips, for early detections of cancers are therefore important and necessary for developing countries.

The total healthcare spending in Vietnam was US $\$ 12.90$ billion in 2014; it is estimated this to reach US $\$ 27.48$ billion in 2020 at a compound annual growth rate of $13.4 \%$ [18]. With the strong support and investments from the government via research and technology development (RTD) funding agencies and projects such as National Foundation for Science and Technology Development (NAFOSTED) and Fostering Innovation through Research, Science and Technology (FIRST), in collaborations with Newton Fund (UK), there are potentials for collaborations in Biomedical Engineering and related areas, especially among research institutions in Vietnam and UK, to develop innovative products and cost-effective solutions for screening, early detections, diagnosis and treatments of cancers for developing countries, including Vietnam.

In conclusions, we presented a microfluidic impedance biosensor which can be used for a real-time monitoring of a single or multiple tumor cells and their response to the anticancer drug treatment. The results of this study open potential research collaborations about development of costeffective devices and lab-on-chips for early disease detection, studies of cancerous cells and their response to anti-cancer drugs to optimize cancer treatments, characterisation of mechanical_properties of cells, new drug delivery mechanisms, and micro \&nano manufacturing. These potential research collaborations may benefit from the currently available RTD resources in micro \& nano manufacturing, nano-materials, and BME, in both Vietnam and UK. For the short and medium term collaborations, based on the successful preliminary results, the results of this study can be expanded to the further test and studies about the effects of the microenvironment on the vital of cells. In addition, we also aim at innovative developments of low-cost microfluidic platforms for developing and testing new antimicrobials on the artificial cells.

\section{ACKNOWLEDGMENT}

British Council - Newton Fund is acknowledged for their support.

\section{REFERENCES}

1 . Giaever and C. R. Keese,(1984) Monitoring fibroblast behavior in tissue culture with an applied electric field. Proceedings of the National Academy of Sciences; 81 (12): 3761-3764

2 Q. Liu,et al. (2014) Cell-based biosensors and their application in biomedicine. Chemical Reviews, 114 (12), 6423-6461

3 F. Asphahani et al. (2007) Cellular impedance biosensors for drug screening and toxin detection. Analyst, 2007: 132: 835-841.

4 I. Giaever and C. R. Keese, (1991) Micromotion of mammalian cells measured electrically. PNAS;:: 88: 7896-7900

5 J. H. Luong et al. (2011) Monitoring motility, spreading, and mortality of adherent insect cells using an impedance sensor. Analytical chemistry; 73 (8): 1844-1848.

6 Q. Liu et al. (2009) Impedance studies of bio-behavior and chemosensitivity of cancer cells by micro-electrode arrays. Biosensors and Bioelectronics; 24 (5): 1305-1310

7 D. Di Carlo (2006) Dynamic single-cell analysis for quantitative biology. Analytical Chemistry; 78 (23): 7918-7925

8 E. K. Sackmann at al. (2014) The present and future role of microfluidics in biomedical research. Nature, 2014: 507 (7491) 181-189

9 H. Yin and D. Marshall (2012) Microfluidics for single cell analysis. Current opinion in biotechnology; 23 (1): 110-119

10 J. Wegener et al. (2000) Electric cell-substrate impedance sensing (ECIS) as a noninvasive means to monitor the kinetics of cell spreading to artificial surfaces. Experimental Cell Research; 259 (1): 158-166.

11 T.A. Nguyen et al. (2013) Microfluidic chip with integrated electrical cell-impedance sensing for monitoring single cancer cell migration in three-dimentional matrixes. Analytical Chemistry; 85 (22): 1106811076

12 B.H. Jo et al. (2000) Three-dimensional micro-channel fabrication in polydimethylsiloxane (PDMS) elastomer. Journal of Microelectromechanical Systems; 9 (1): 76-81

13 T. Anh-Nguyen et al. (2016) An impedance biosensor for monitoring cancer cell attachment, spreading and drug-induced apoptosis. Sensors and Actuators A: Physical; 241: 231-237

14 J. Ferlay et al. (2012) Cancer incidence and mortality worldwide: sources, methods and major patterns in GLOBOCAN 2012. Int. J. Cancer 136 (5): e359-e386

15. Jemal A. et al. (2011) Global cancer statistics. CA: A Cancer Journal for Clinicians; 61(2):69-90

16. Ramaiah V. K. et al. (2015) Health-care related supportive-care factors may be responsible for poorer survival of cancer patients in developing countries. Journal of Cancer Policy; 5:31-47

17. Miriam L.G. et al. (2013) Cancer in developing countries: The next most preventable pandemic. The Global problem of cancer. Critical Reviews in Oncology/Hematology; 88:117-122

18. Vietnam Healthcare Outlook: www.frost.com [Access: April 2016]

Author: Tien Anh Nguyen

Institute: Le Quy Don Technical University

Address: 236 Hoang Quoc Viet Street, Bac Tu Liem District

City: $\quad$ Ha Noi

Country: Viet Nam

Email: anh.nguyen@imtek.de or anhnt007@gmail.com 